\title{
Naringenin attenuates pressure overload-induced cardiac hypertrophy
}

\author{
NING ZHANG ${ }^{1,2^{*}}$, ZHENG YANG $^{1,2^{*}}$, YUAN YUAN $^{1,2}$, FANGFANG LI $^{1,2}$, YUAN LIU ${ }^{1,2}$, ZHENGUO MA $^{1,2}$, \\ HAIHAN LIAO $^{1,2}$, ZHOUYAN BIAN ${ }^{1,2}$, YAO ZHANG ${ }^{1,2}$, HENG ZHOU $^{1,2}$, WEI DENG ${ }^{1,2}$, \\ MENGQIAO ZHOU ${ }^{1,2}$ and QIZHU TANG ${ }^{1,2}$ \\ ${ }^{1}$ Department of Cardiology, Renmin Hospital of Wuhan University; ${ }^{2}$ Cardiovascular Research Institute, \\ Wuhan University, Wuhan, Hubei 430060, P.R. China
}

Received September 29, 2014; Accepted September 1, 2015

DOI: $10.3892 /$ etm.2015.2816

\begin{abstract}
Cardiac hypertrophy is characterized by abnormal enlargement of cardiomyocytes and disproportionate accumulation of extracellular interstitial fibrosis, which are major predictors of the development of coronary artery disease and heart failure. Naringenin is a bitter principle component of grapefruit that has numerous pharmacological effects, including anti-inflammatory, hypolipidemic, antithrombotic and antiatherogenic properties. In order to investigate whether naringenin is able to exert a protective effect against cardiac hypertrophy induced by pressure overload, aortic banding (AB) was performed to induce cardiac hypertrophy in mice, and naringenin was administered for 7 weeks. A total of 60 mice were allocated into four groups: Sham + vehicle, $A B+$ vehicle, sham + naringenin and $A B+$ naringenin. Naringenin treatment attenuated cardiac dysfunction, as indicated by the results of echocardiography and catheter-based measurements at 8 weeks post-surgery. The extent of cardiac hypertrophy was assessed by the heart weight/body weight, heart weight/tibial length and lung weight/body weight ratios, in addition to the cardiomyocyte cross-sectional area and the mRNA expression levels of hypertrophic maker, all of which were mitigated by naringenin administration. Naringenin also inhibited the expression of transforming growth factor- $\beta 1$, connective tissue growth factor, collagen I $\alpha$ and collagen III $\alpha$, and attenuated interstitial fibrosis. In addition, naringenin downregulated the activation of the extracellular signal-regulated kinase (ERK), c-Jun N-terminal kinase (JNK) and phosphoinositide 3-kinase $(\mathrm{PI} 3 \mathrm{~K}) /$ protein kinase $\mathrm{B}(\mathrm{Akt})$ signaling pathways. In
\end{abstract}

Correspondence to: Professor Qizhu Tang, Department of Cardiology, Renmin Hospital of Wuhan University, 238 Jiefang Road, Wuhan, Hubei 430060, P.R. China

E-mail: qztang@whu.edu.cn

*Contributed equally

Key words: cardiac hypertrophy, naringenin, aortic banding, pharmacology conclusion, naringenin attenuated cardiac hypertrophy and interstitial fibrosis, in addition to improving left ventricular function in pressure-overloaded mice. The cardioprotective effect exerted by naringenin may be associated with the inhibition of PI3K/Akt, ERK and JNK signaling pathways.

\section{Introduction}

Pathological cardiac hypertrophy develops in response to hemodynamic overload, which is a major predictor for the development of coronary artery disease and heart failure (1). Preventing and postponing the progression of cardiac hypertrophy may serve as an effective therapeutic strategy for the treatment of patients with heart failure (2). Clinical management of pathological cardiac hypertrophy is targeted against the underlying cause of the condition and typically involves the administration of certain pharmacological agents, such as angiotensin-converting-enzyme inhibitor and angiotensin receptor blockers (3). However, although current pharmacological approaches appear to be beneficial in improving the quality of life of patients with heart failure, they do not markedly reduce the mortality rates (4). A previous study showed that puerarin attenuates the cardiac hypertrophy induced by pressure overload by downregulating phosphoinositide 3-kinase (PI3K)/protein kinase B (Akt) and c-Jun $\mathrm{N}$-terminal kinase (JNK) signaling pathways (5). Therefore, a key future challenge is the identification of pharmacological agents that alleviate progressive myocardial dysfunction and unfavorable remodeling, improve the quality of life and reduce the mortality rate of patients with heart failure.

Naringenin is a bitter principle component of grapefruit (Citrus paradisi) that exhibits a variety of pharmacological effects, including anti-inflammatory (6), hypolipidemic, antithrombotic and antiatherogenic properties (5). The dietary consumption of citrus fruits is associated with a reduced rate of acute coronary events (7), and naringenin appears to exert beneficial effects on the cardiovascular system (8). Naringenin has been demonstrated to promote endothelium-independent vasorelaxing effects on rat aortic rings that have been precontracted with noradrenaline (9). Furthermore, naringenin may be able to alleviate myocardial ischemia/reperfusion injury (10). In addition, due to its radical-scavenging and 
iron-chelating properties, naringenin may potentially protect against doxorubicin-induced cardiac toxicity (6). However, it currently remains clear whether naringenin is able to mitigate the development of pressure overload-induced cardiac hypertrophy and postpone the progression of heart failure.

In the present study, aortic banding (AB) was used to induce cardiac hypertrophy in $\mathrm{C} 57 \mathrm{BL} / 6$ mice in order to investigate whether naringenin is able to exert a protective effect against cardiac hypertrophy, fibrosis and left ventricular dysfunction, and to identify the possible mechanisms underlying these effects. In addition, the study aimed to determine whether naringenin is able to influence the activation of mitogen-activated protein kinase (MAPK) and PI3K/Akt signaling pathways, which are activated by pressure overload.

\section{Materials and methods}

Animals and animal models. All the experimental procedures were performed in accordance with the institutional guidelines of the Animal Care and Use Committee of Renmin Hospital of Wuhan University (Wuhan, China) and in accordance with the Guide for the Care of Laboratory Animals published by the US National Institutes of Health (NIH publication no. 85-23, revised in 1996). This study was approved by the ethics committee of Wuhan University (Wuhan, China). A total of 60 male C57BL/6 mice (age, 8-10 weeks; weight, 23.5-27.5 g) were purchased from the Institute of Laboratory Animal Science, CAMS \& PUMC (Beijing, China). All animals were allowed to acclimatize to the laboratory environment for 1 week, then randomly distributed to either a sham surgery or AB group, which were respectively treated without or with naringenin (normal diet containing $\sim 100 \mathrm{mg} / \mathrm{kg}$ body weight/day naringenin) for 7 weeks, beginning 1 week after AB surgery. The dose of naringenin was administered according to the protocol described in a previous study (10). Non-naringenin (vehicle) mice received a normal diet of rodent chow. Subsequently, the C57BL/6 mice were allocated at random into four groups: Sham + naringenin, $\mathrm{AB}+$ naringenin, sham + vehicle and $A B+$ vehicle ( $n=15$ per group). $A B$ was performed as described previously (11). At 8 weeks after surgery, the animals were euthanized by cervical dislocation while anesthetized with $1.5 \%$ isoflurane, and the hearts were dissected and weighed to calculate the heart weight/body weight (HW/BW, in $\mathrm{mg} / \mathrm{g})$, heart weight/tibia length (HW/TL, in $\mathrm{mg} / \mathrm{mm}$ ) and lung weight/body weight (LW/BW, in $\mathrm{mg} / \mathrm{g}$ ) ratios in the naringenin-treated and vehicle-treated mice. All surgeries and analyses were performed in a blinded manner.

Echocardiography and hemodynamics. Transthoracic echocardiography and hemodynamic analysis were performed according to the protocol described in a previous study (12). The animals were subjected to echocardiographic and pressure-volume analyses at 8 weeks after surgery. For echocardiography measurements, mice was anesthetized with $1.5 \%$ isoflurane, and measurements were obtained using a MyLab $^{\text {TM }}$ 30CV ultrasound system (Esaote SpA, Genoa, Italy) equipped with a $10-\mathrm{MHz}$ linear array ultrasound transducer. The left ventricle (LV) dimensions were assessed in a parasternal short-axis view during systole or diastole. The LV end-systolic diameter (LVESD), LV end-diastolic diameter (LVEDD) and wall thickness were obtained from the smallest or largest area of the LV. In the process of hemodynamic measurements, a microtip catheter transducer (Millar, Inc., Houston, TX, USA) was inserted into the right carotid artery and advanced into the LV of mice anesthetized with $1.5 \%$ isoflurane. The signals were continuously recorded using a Millar Pressure-Volume system (Millar, Inc.), and the maximal rate of pressure development $\left(\mathrm{dP} / \mathrm{dt}_{\max }\right)$ and minimal rate of pressure decay $\left(\mathrm{dP} / \mathrm{dt}_{\text {min }}\right)$ were processed using PVAN data analysis software (Millar, Inc.).

Histological analysis. The hearts of the mice were excised, arrested in diastole with $10 \% \mathrm{KCl}$, weighed, placed in $10 \%$ formalin and embedded in paraffin. The hearts were cut transversely close to the apex to visualize the left and right ventricles. Sections of the heart $(4-5 \mathrm{~mm})$ were obtained and mounted onto slides, stained with hematoxylin and eosin (H\&E; Baso Diagnostics, Inc., Zhuhai, China) for histopathological analysis. For collagen deposition determination, tissue sections were stained with $0.1 \%$ picro-sirius red (PSR) red satin solution (Dechuang, Inc., Beijing, China) for $1 \mathrm{~h}$ at room temperature, then washed in $0.5 \%$ acetic acid for $2 \mathrm{~min}$ and visualized by light microscopy (ECLIPSE 80i; Nikon Corporation, Tokyo, Japan). For myocyte cross-sectional area examination, sections were stained with H\&E. A single myocyte per field was observed using the Image-Pro Plus 6.0 quantitative digital image analysis system (Media Cybernetics, Inc., Rockville, MD, USA). The outline of 100-200 myocytes in the LV was analyzed for each group.

Reverse transcription-quantitative polymerase chain reaction (RT-qPCR) and western blot analysis. In order to investigate the relative mRNA expression levels of atrial natriuretic peptide (ANP), B-type natriuretic peptide (BNP), $\beta$-myosin heavy chain ( $\beta$-MHC), transforming growth factor- $\beta$ (TGF- $\beta$ ), connective tissue growth factor (CTGF), collagen I $\alpha$ and collagen III $\alpha$, total mRNA was extracted from frozen, pulverized LV tissue using TRIzol reagent (cat. no. 15596026; Invitrogen Life Technologies, Carlsbad, CA, USA). cDNA was synthesized from $2 \mathrm{mg}$ total RNA using oligo (dT) primers and an RT-for-PCR kit (cat. no. 04896866001; Roche Diagnostics $\mathrm{GmbH}$, Mannheim, Germany). PCR amplifications were performed using a LightCycler 480 SYBR Green Master Mix (cat. no. 04896866001; Roche Diagnostics GmbH). The cycling conditions for PCR were as follows: $10 \mathrm{sec}$ denaturation at $95^{\circ} \mathrm{C}, 20 \mathrm{sec}$ annealing at $60^{\circ} \mathrm{C}$ and $20 \mathrm{sec}$ at $72^{\circ} \mathrm{C}$ extension. The results were normalized against the expression levels of glyceraldehyde-3-phosphate dehydrogenase (GAPDH).

In order to determine the activation state of MAPK and PI3K/Akt signaling, cardiac tissues were lysed in RIPA lysis buffer. Subsequently, the protein concentration of all samples was measured using a bicinchoninic assay kit (cat. no. 23227; Thermo Fisher Scientific, Waltham, MA, USA). Tissue lysate samples $(50 \mathrm{mg}$ ) were subjected to $10 \%$ SDS-PAGE, and subsequently transferred to polyvinylidene difluoride membranes (cat. no. IPFL00010; EMD Millipore, Billerica, MA, USA). The membranes were blocked with $5 \%$ non-fat milk and then incubated overnight at $4{ }^{\circ} \mathrm{C}$ with the following rabbit primary antibodies against: Phosph-PI3K p85Tyr458/p55Tyr199 (4288), total-PI3K p85 (4257), phospho-AktSer473 (4060), total-Akt 

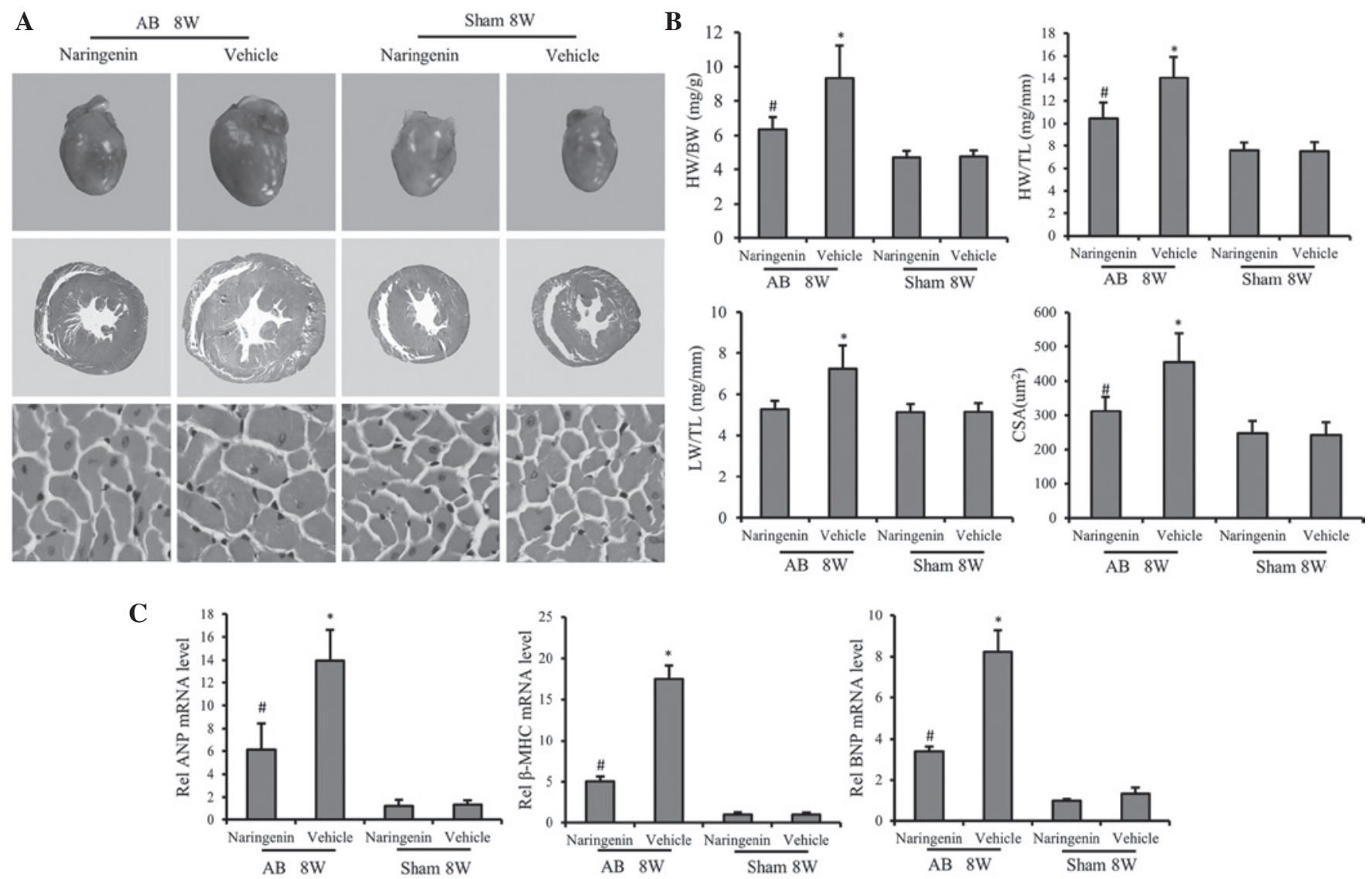

Figure 1. Effect of naringenin on cardiac hypertrophy. (A) Gross hearts (top), whole heart section (middle; magnification, x10) and cardiomyocyte cross-sectional area (bottom; magnification, x400) stained with hematoxylin and eosin at 8 weeks after AB surgery. Naringenin treatment alleviated cardiac hypertrophy and decreased the cardiomyocyte cross-sectional area following AB surgery. (B) Results of the HW/BW, LW/BW and HW/TL ratios, and myocyte CSA of the indicated groups. (C) Expression of transcripts for ANP, BNP and $\beta$-MHC of the indicated groups were determined by reverse transcription-polymerase chain reaction analysis (fold changes). ${ }^{*} \mathrm{P}<0.05$ vs. corresponding sham group. ${ }^{\prime} \mathrm{P}<0.05$ vs. $\mathrm{AB}+$ vehicle group. $\mathrm{AB}$, aortic banding; $8 \mathrm{~W}, 8$ weeks; $\mathrm{ANP}$, atrial natriuretic peptide; $\beta$-MHC, $\beta$-myosin heavy chain; BNP, B-type natriuretic peptide; HW, heart weight; BW, body weight; TL, tibial length; CSA, cross-sectional area.

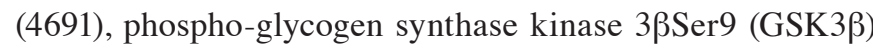
(9322), total-GSK3 $\beta$ (9315), phospho-extracellular signal-regulated kinase (ERK)1/2Thr202/Tyr204 (4370), total-ERK1/2 (4695), phospho-p38Thr180/Tyr182 (4511), total-p38 (9212), phospho-JNK1/2Thr183/Tyr18 (4668), total-JNK1/2 (9285) (1:1,000; Cell Signaling Technology, Inc., Danvers, MA, USA). In addition, rabbit anti-GAPDH antibody (1:1,000; MB001) was purchased from Bioworld Technology, Inc. (St. Louis Park, MN, USA). The blots were then scanned using a two-color infrared imaging system (Odyssey; LI-COR Biosciences, Lincoln, NE, USA). Specific protein expression levels were normalized against those of GAPDH protein for total tissue lysates.

Statistical analysis. Data are expressed as the mean \pm standard deviation. Differences among groups were determined by two-way analysis of variance followed by a post-hoc Tukey's test. Comparisons between two groups were performed using an unpaired Student's t-test. $\mathrm{P}<0.05$ was considered to indicate a statistically significant difference.

\section{Results}

Naringenin protects against pressure overload-induced cardiac hypertrophy. To determine the role of naringenin in cardiac remodeling, hypertrophic responses were evaluated using histological analysis and RT-qPCR. H\&E staining of gross heart tissue confirmed the protective effect of naringenin against cardiac hypertrophy. $\mathrm{H} \& \mathrm{E}$ staining of gross heart tissue indicated that the increase in the cardiomyocyte cross-sectional area following $\mathrm{AB}$ was inhibited by naringenin (Fig. 1A). At 8 weeks of $A B$ treatment, mice in the $A B+$ vehicle group exhibited significant increases in the HW/BW, HW/TL and LW/BW ratios compared with the sham + vehicle group. Administration of naringenin evidently decreased the HW/BW and HW/TL ratios following $\mathrm{AB}$ $(\mathrm{P}<0.05)$, indicating the attenuation of cardiac hypertrophy; however, no statistically significant difference was detected in the LW/BW ratio ( $\mathrm{P}>0.05)$ (Fig. 1B). Furthermore, in mice subjected to $A B$, the mRNA expression levels of hypertrophic markers, including ANP, BNP and $\beta$-MHC, was mitigated by naringenin, as determined by RT-qPCR $(\mathrm{P}<0.05)$ (Fig. 1C).

Naringenin improves impaired cardiac function following $A B$. To evaluate the effect of naringenin in the progression of cardiac dysfunction, $\mathrm{AB}$ or sham surgery was performed. After 8 weeks, the chamber diameter, wall thickness and function of the LV were assessed by echocardiography. No statistically significant differences were detected between 
A

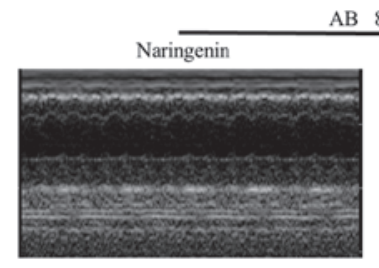

B
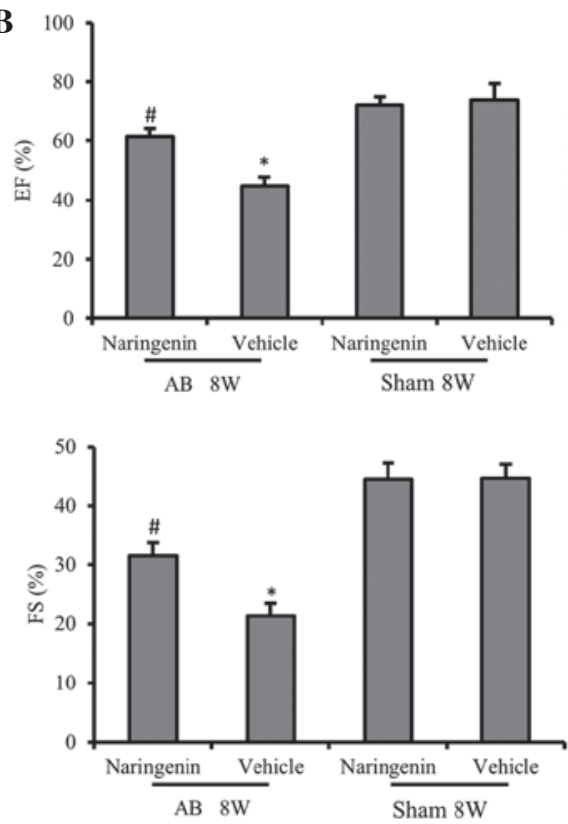

$8 \mathrm{~W}$
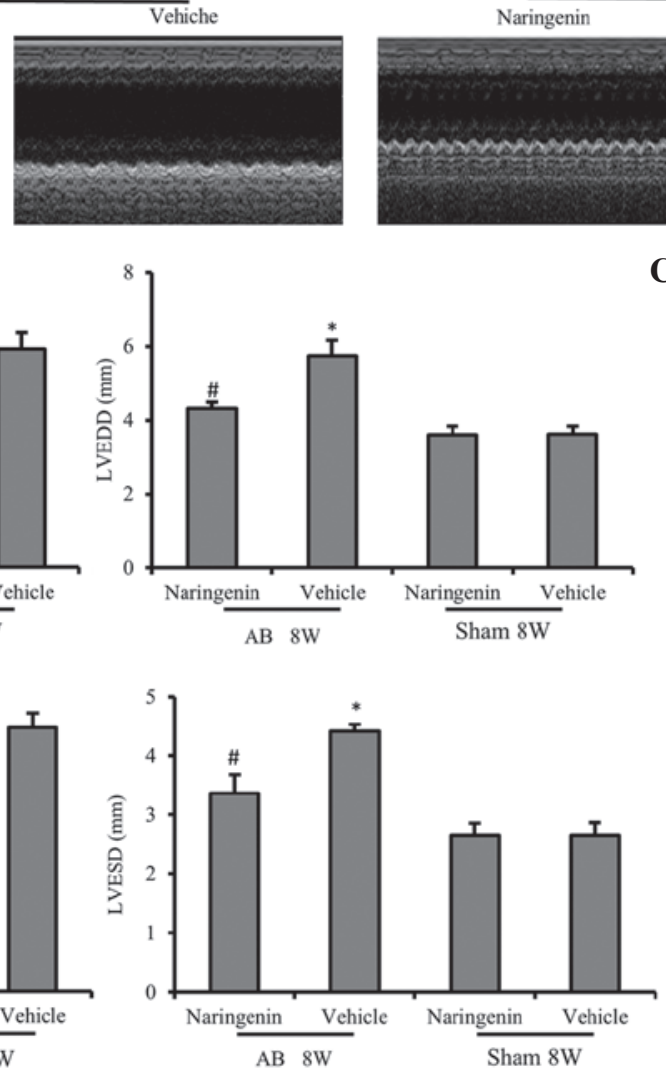

C
Sham 8W
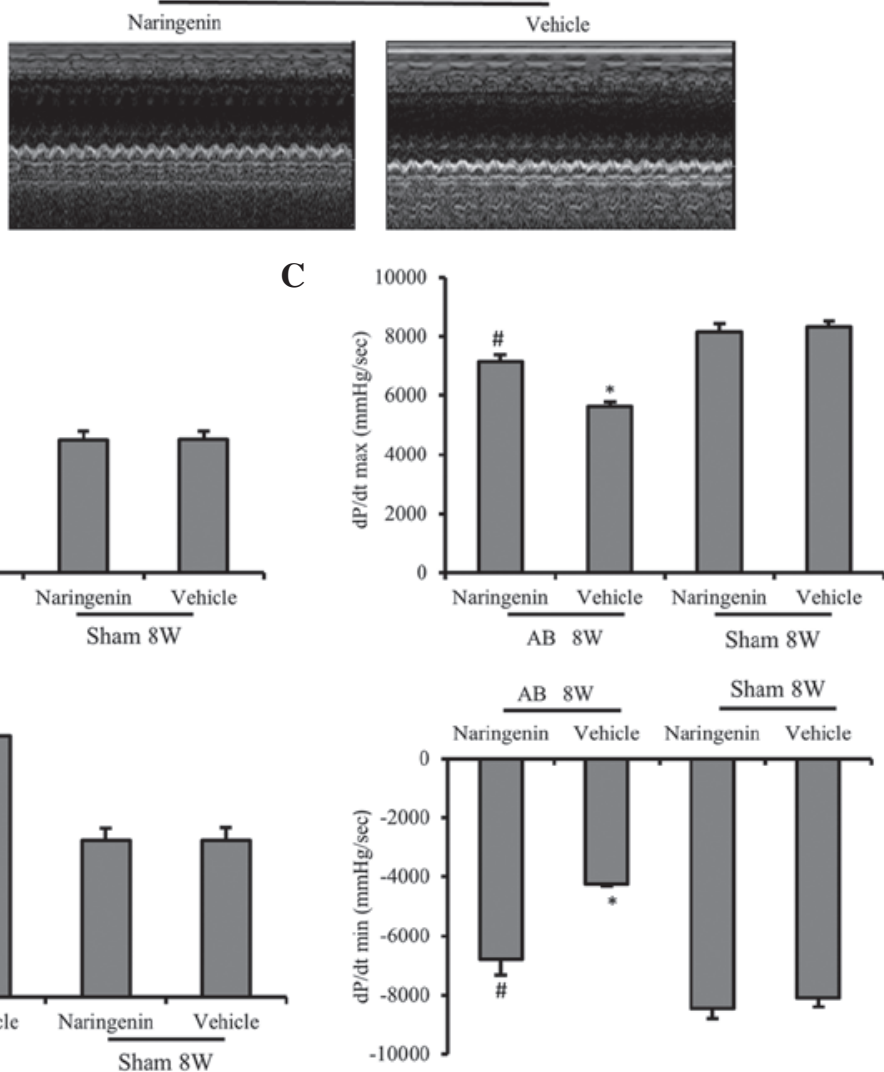

Figure 2. Echocardiography and pressure-volume analysis results indicating that naringenin improves the cardiac function. (A) Representative M-mode images of AB and sham groups. (B) Naringenin attenuated pressure overload-induced increased LV diameters, including the LVESD, LVEDD, FS and EF. (C) Cardiac function data of hemodynamic parameters including $\mathrm{dp} / \mathrm{dt}_{\max }$ and $\mathrm{dp} / \mathrm{dt}_{\min } .{ }^{*} \mathrm{P}<0.05 \mathrm{vs}$. corresponding sham group. ${ }^{\#} \mathrm{P}<0.05 \mathrm{vs}$. $\mathrm{AB}+$ vehicle group. $\mathrm{AB}$, aortic banding; EF, ejection fraction; LVEDD, left ventricular (LV) end-diastolic diameter; LVESD, LV end-systolic diameter; FS, fractional shortening; dp/dt ${ }_{\text {max }}$, maximal rate of pressure development; $\mathrm{dp} / \mathrm{dt}_{\min }$, minimal rate of pressure decay.

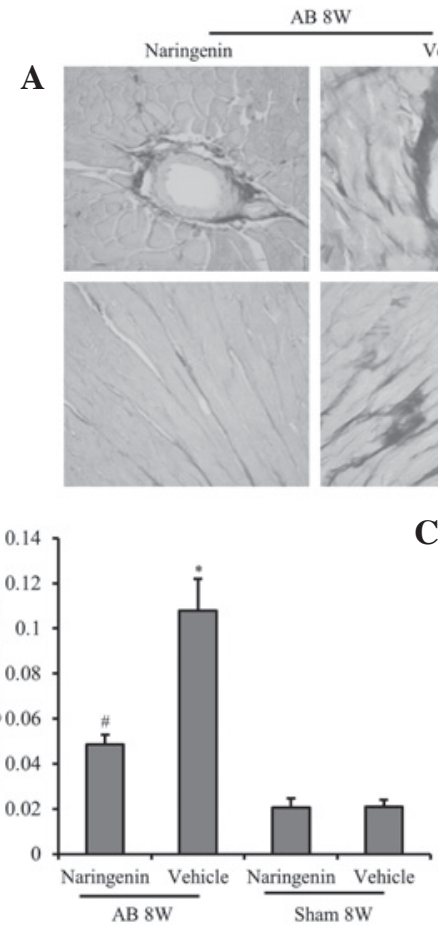

vehicle
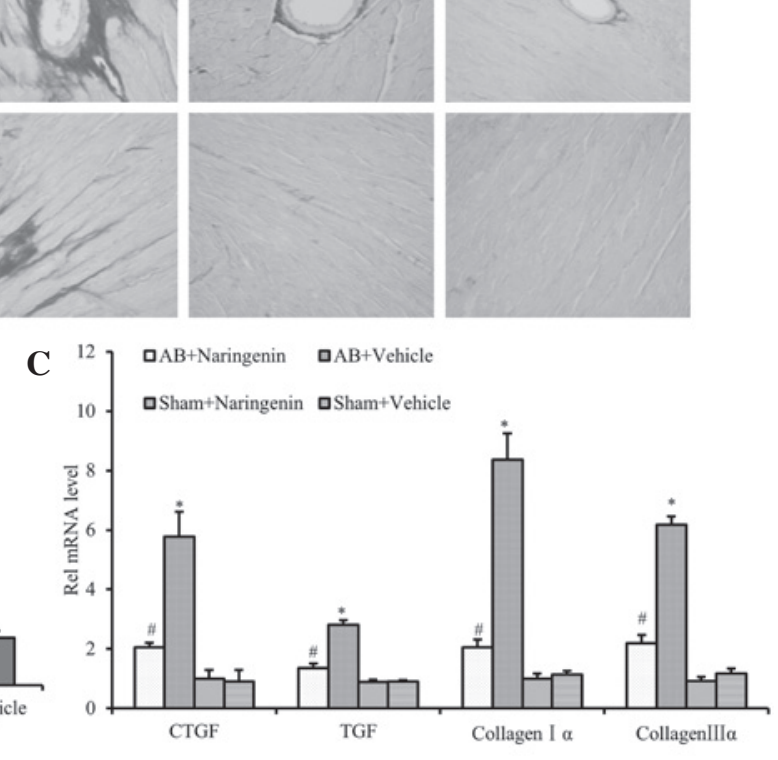

Figure 3. Naringenin attenuates cardiac interstitial fibrosis subsequent to pressure overload. (A) Cardiac interstitial fibrosis determined in heart sections of the indicated groups by picrosirius red staining, naringenin treatment decreased cardiac interstitial fibrosis induced by pressure-overload after $\mathrm{AB}$ surgery (x100). (B) Fibrotic areas ratio of histological sections were quantified by an image-analysis system. (C) mRNA expression levels of TGF- $\beta 1$, CTGF, collagen I $\alpha$ and collagen III $\alpha$ in the myocardium of the indicated groups, determined using reverse transcription-polymerase chain reaction analysis. "P<0.05 vs. corresponding sham group. ${ }^{\#} \mathrm{P}<0.05$ vs. $\mathrm{AB}+$ vehicle group. $\mathrm{AB}$, aortic banding; $\mathrm{LV}$, left ventricular; $\mathrm{CTGF}$, connective tissue growth factor; TGF- $\beta 1$, transforming growth factor- $\beta 1$. 

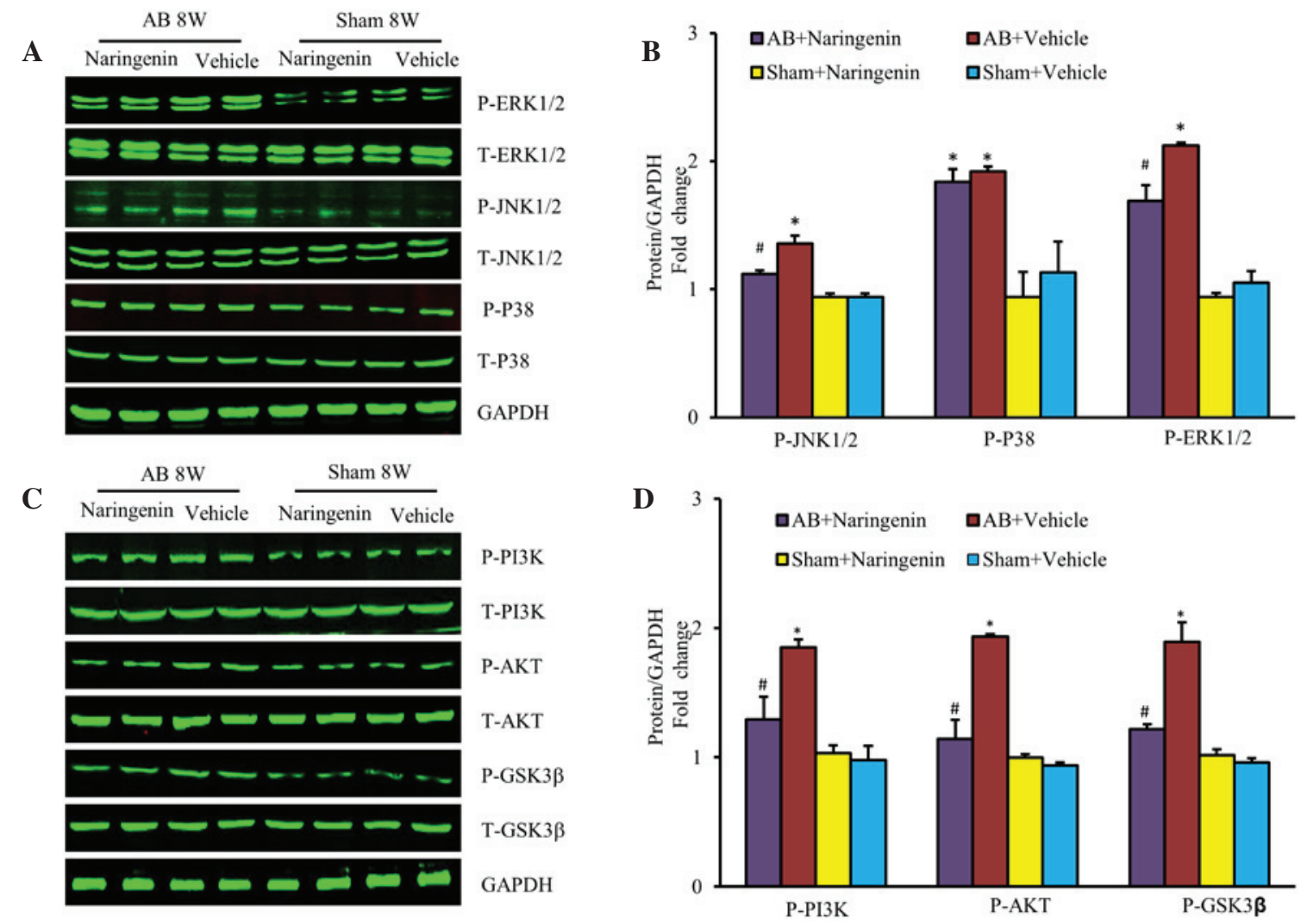

Figure 4. Effects of naringenin on mitogen-activated protein kinases and PI3K/AKT signalling in response to hypertrophic stimuli. (A) Representative and (B) quantitative expression of phosphorylated and total ERK1/2, JNK1/2, p38, and the effects of naringenin on indicated groups. (C) Representative and (D) quantitative blots of phosphorylated and total PI3K, Akt, GSK3 $\beta$ in the heart tissues of mice in the indicated groups, with GAPDH used as the loading control. " $\mathrm{P}<0.05$ vs. corresponding sham group. ${ }^{\#} \mathrm{P}<0.05$ vs. $\mathrm{AB}+$ vehicle group. $\mathrm{AB}$, aortic banding; ERK, extracellular signal-regulated kinase; JNK, c-Jun

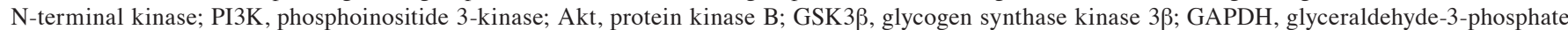
dehydrogenase.

the vehicle and naringenin-treated mice that underwent the sham surgery. However, following AB surgery, the naringenin-treated mice exhibited alleviated cardiac hypertrophy and cardiac dysfunction compared with the vehicle mice, which was determined by measuring the LVESD, LVEDD, fractional shortening and ejection fraction $(\mathrm{P}<0.05)$ (Fig. 2A and B). The $\mathrm{dP} / \mathrm{dt}_{\max }$ and $\mathrm{dP} / \mathrm{dt}_{\min }$, analyzed by pressure-volume loop, revealed that the naringenin treatment exerted a beneficial effect on the hemodynamic function of LV (Fig. 2C).

Naringenin alleviates the fibrotic response induced by pressure overload. To clarify the effects of naringenin on perivascular and interstitial fibrosis, cardiac interstitial collagen deposition was detected using PSR staining. The results demonstrated that the fibrotic area ratio was decreased significantly in mice that received naringenin treatment following $A B$ surgery $(\mathrm{P}<0.05)$. However, there was no significant difference in the extent of cardiac fibrosis in the sham-operated mice after 8 weeks $(\mathrm{P}>0.05)$ (Fig. 3A and B). To further elucidate the effect of naringenin on collagen synthesis, the mRNA expression levels of TGF- $\beta$, CTGF, collagen I $\alpha$ and collagen III $\alpha$, which are known mediators of fibrosis, were analyzed. Compared with the vehicle group, decreased expression levels of TGF- $\beta$, CTGF, collagen I $\alpha$ and collagen III $\alpha$ were detected in the naringenin group following the $\mathrm{AB}$ surgery $(\mathrm{P}<0.05)$ (Fig. 3C).
Naringenin attenuates cardiac hypertrophy by inhibiting the activation of JNK, ERK and PI3K/Akt signaling pathways. To elucidate the molecular mechanisms by which naringenin mediates cardiac hypertrophy, the activation states of the MAPK and PI3K/Akt signaling pathways were evaluated. The results indicated that the upregulation of phospho-JNK and phospho-ERK was mitigated in mice that received naringenin treatment following the $\mathrm{AB}$ surgery $(\mathrm{P}<0.05)$. However, the level of phospho-p38 was unchanged ( $P>0.05$ ) (Fig. 4A and B). Furthermore, the role of the PI3K/Akt signaling pathway, a key mechanism that regulates the progression of pathological cardiac hypertrophy, was investigated. Naringenin also appeared to decrease the expression of phosphorylated Akt and its downstream target phospho-GSK3 $\beta$ subsequent to pressure overload $(\mathrm{P}<0.05)$ (Fig. $4 \mathrm{C}$ and $\mathrm{D})$.

\section{Discussion}

Naringenin is widely used in traditional Chinese medicine due to its multiple pharmacological effects, including anti-inflammatory (5), anti-hyperlipidemic, anti-thrombotic and anti-atherosclerotic properties. Naringenin promotes endothelium-independent vasorelaxing effects on rat aortic rings pre-contracted with noradrenaline (9), and is able to alleviate the myocardial ischemia/reperfusion injury (10). A previous study reported that naringenin is able to protect against doxorubicin-induced cardiac toxicity, due to its 
radical-scavenging and iron-chelating properties (6). To the best of our knowledge, the present study demonstrated for the first time that naringenin is able to attenuate pressure overload-induced cardiac hypertrophy. Furthermore, the results indicated that naringenin may improve cardiac function and attenuate the interstitial fibrosis.

Cardiac hypertrophy is characterized by the abnormal enlargement of cardiomyocytes, originating from an increase in myocyte size and the proliferation of non-muscle cells (13). Numerous clinical studies have indicated that sustained cardiac hypertrophy is a maladaptive process, ultimately leading to decompensated heart failure (14). Characterization of the pathogenesis of cardiac hypertrophy may provide novel approaches for the prevention of heart failure progression. Blockage of the MAPK signaling pathway, downstream of which a variety of intracellular transcription factors are phosphorylated during the reprogramming of cardiac gene expression, prevents the progression of cardiac hypertrophy (15). In addition, the ERK pathway serves a critical function in cardiac hypertrophy, and may be activated by mitogen-activated protein kinase kinase (known as MEK or MAPKK)-dependent phosphorylation (16). JNKs are specific transducers of the stress response, and are known as stress-activated protein kinases (17). In the heart, JNK activity is upregulated following pressure overload (18). However, Lei et al (19) reported that the expression of p38 MAPK was upregulated transiently in transverse AB-induced pressure overload in mice. However, the molecular mechanism by which naringenin mitigates cardiac hypertrophy remain unclear. Therefore, in the present study the activation of potential signaling pathways, including the MAPK and PI3K/Akt pathways, was evaluated. The results of the present study demonstrate that naringenin is able to mitigate the activation of the ERK and JNK signaling pathways. PI3K/Akt is another crucial signaling pathway in the process of cardiac hypertrophy. Various studies have indicated that the engagement of the p85SH2 domains of PI3K by pTyr relieved the p85-mediated inhibition of p110 isoforms, led to the activation of class I PI3Ks (p110 $\alpha, \mathrm{p} 110 \delta, \mathrm{p} 110 \beta)$ and always resulted in Akt activation (20). The short-term activation of Akt known to be caused by physiological hypertrophy and through such pathways may be cardioprotective, despite causing mild heart enlargement; however, the long-term activation promoted pathological cardiac hypertrophy and heart failure (21). Inhibition of GSK3 $\beta$, downstream of Akt, is required for compensation of pressure overload (22), and has been shown to be a negative regulator of cardiac hypertrophy (23). Notably, naringenin attenuates the activation of Akt in the progression of cardiac hypertrophy.

Fibrosis is another integral feature of cardiac hypertrophy, which is characterized by the disproportionate expression of the extracellular matrix and accumulation of fibrillar collagen. The primary collagen types in the heart are type I and III, which together account for $>90 \%$ of the total collagen and are the predominant contributors to interstitial fibrosis in the progression of heart failure (24). The present results indicate that the mRNA expression levels of TGF- $\beta 1$, CTGF, collagen I $\alpha$ and collagen III $\alpha$ were increased following $\mathrm{AB}$, and that naringenin evidently alleviated this upregulation. In addition, the results of the PSR staining suggested that naringenin attenuates the interstitial and perivascular fibrosis induced by pressure overload (25). Notably, naringenin has the pharmacological effect of normalizing the expression of pro-fibrotic genes, indicating that naringenin may be effective in attenuating extracellular remodeling following pressure overload. These effects require verification by additional studies.

In conclusion, the results of the present study demonstrated that naringenin exerts a pharmacological effect against the progression of cardiac hypertrophy induced by pressure overload. Inhibition of the JNK, ERK and PI3K/Akt signaling pathways by naringenin may serve a crucial function in the mitigation of the development of cardiac hypertrophy in structure remodeling and function alteration induced by pressure overload. This anti-fibrotic function is mediated by the downregulation of interstitial fibrosis gene expression, including that of TGF- $\beta 1$, CTGF, collagen I $\alpha$ and collagen III $\alpha$, which occur in response to pressure overload. These results partially elucidate the pharmacological effects of naringenin and the pathways involved in its protective role, which may provide a novel pharmacotherapeutic strategy for the treatment of cardiac hypertrophy in pressure overload and limit the progression of heart failure.

\section{Acknowledgements}

This study was supported by grants from the National Natural Science Foundation of China (no. 81270303 and 81300104), the Specialized Research Fund for the Doctoral Program of Higher Education of China (no. 20130141120042) and the Natural Science Foundation of Hubei Province, China (no. 2013CFB303).

\section{References}

1. Balakumar P and Jagadeesh G: Multifarious molecular signaling cascades of cardiac hypertrophy: Can the muddy waters be cleared? Pharmacol Res 62: 365-383, 2010.

2. Gjesdal O, Bluemke DA and Lima JA: Cardiac remodeling at the population level-risk factors, screening and outcomes. Nat Rev Cardiol 8: 673-685, 2011.

3. Hellawell JL and Margulies KB: Myocardial reverse remodeling. Cardiovasc Ther 30: 172-181, 2012.

4. van Berlo JH, Maillet M and Molkentin JD: Signaling effectors underlying pathologic growth and remodeling of the heart. J Clin Invest 123: 37-45, 2013

5. Yuan Y, Zong J, Zhou H, Bian ZY, Deng W, Dai J, Gan HW, Yang Z, Li H and Tang QZ: Puerarin attenuates pressure overload-induced cardiac hypertrophy. J Cardiol 63: 73-81, 2014.

6. Middleton E Jr and Kandaswami C: Effects of flavonoids on immune and inflammatory cell functions. Biochem Pharmacol 43: 1167-1169, 1992.

7. Oshipura KJ, Hu FB, Manson JE, Stampfer MJ, Rimm EB, Speizer FE, Colditz G, Ascherio A, Rosner B, Spiegelman D and Willett WC: The effect of fruit and vegetable intake on risk for coronary heart disease. Ann Intern Med 134: 1106-1114, 2001.

8. Dauchet L, Ferrières J, Arveiler D, Yarnell JW, Gey F, Ducimetière P, Ruidavets JB, Haas B, Evans A, Bingham A, et al: Frequency of fruit and vegetable consumption and coronary heart disease in France and Northern Ireland: The PRIME study. Br J Nutr 92: 963-972, 2004.

9. Saponara S, Testai L, Iozzi D, Martinotti E, Martelli A, Chericoni S, Sgaragli G, Fusi F and Calderone V: (+/-)-Naringenin as large conductance $\mathrm{Ca}(2+)$-activated $\mathrm{K}+(\mathrm{BKCa})$ channel opener in vascular smooth muscle cells. Br J Pharmacol 149: 1013-1021, 2006.

10. Testai L, Martelli A, Marino A, D'Antongiovanni V, Ciregia F, Giusti L, Lucacchini A, Chericoni S, Breschi MC and Calderone V: The activation of mitochondrial BK potassium channels contributes to the protective effects of naringenin against myocardial ischemia/reperfusion injury. Biochem Pharmacol 85: 1634-1643, 2013. 
11. Yan L, Huang H, Tang QZ, Zhu LH, Wang L, Liu C, Bian ZY and $\mathrm{Li} \mathrm{H}$ : Breviscapine protects against cardiac hypertrophy through blocking PKC-alpha-dependent signaling. J Cell Biochem 109: $1158-1171,2010$.

12. Zhou H, Bian ZY, Zong J, Deng W, Yan L, Shen DF, Guo H, Dai J, Yuan Y, Zhang R, et al: Stem cell antigen 1 protects against cardiac hypertrophy and fibrosis after pressure overload. Hypertension 60: 802-809, 2012.

13. Carreño JE, Apablaza F, Ocaranza MP and Jalil JE: Cardiac hypertrophy: Molecular and cellular events. Rev Esp Cardiol 59: 473-486, 2006 (In Spanish).

14. Frey $\mathrm{N}$ and Olson EN: Cardiac hypertrophy: The good, the bad and the ugly. Annu Rev Physiol 65: 45-79, 2003.

15. Lorenz K, Schmitt JP, Vidal M and Lohse MJ: Cardiac hypertrophy: Targeting Raf/MEK/ERK1/2-signaling. Int J Biochem Cell Biol 41: 2351-2355, 2009.

16. Lorenz K, Schmitt JP, Schmitteckert EM and Lohse MJ: A new type of ERK1/2 autophosphorylation causes cardiac hypertrophy. Nat Med 15: 75-85, 2009.

17. Paul A, Wilson S, Belham CM, Robinson CJ, Scott $\mathrm{PH}$ Gould GW and Plevin R: Stress-activated protein kinases: Activation, regulation and function. Cell Signal 9: 403-410, 1997

18. Sopontammarak S, Aliharoob A, Ocampo C, Arcilla RA Gupta MP and Gupta M: Mitogen-activated protein kinases (p38 and c-Jun NH2-terminal kinase) are differentially regulated during cardiac volume and pressure overload hypertrophy. Cell Biochem Biophys 43: 61-76, 2005.
19. Lei B, Chess DJ, Keung W, O'Shea KM, Lopaschuk GD and Stanley WC: Transient activation of p38 MAP kinase and up-regulation of Pim-1 kinase in cardiac hypertrophy despite no activation of AMPK. J Mol Cell Cardiol 45: 404-410, 2008.

20. Vanhaesebroeck B, Guillermet-Guibert J, Graupera M and Bilanges B: The emerging mechanisms of isoform-specific PI3K signalling. Nat Rev Mol Cell Biol 11: 329-341, 2010.

21. Chaanine $\mathrm{AH}$ and Hajjar RJ: AKT signalling in the failing heart. Eur J Heart Fail 13: 825-829, 2011.

22. Badorff C, Ruetten H, Mueller S, Stahmer M, Gehring D, Jung F, Ihling C, Zeiher AM and Dimmeler S: Fas receptor signaling inhibits glycogen synthase kinase 3 beta and induces cardiac hypertrophy following pressure overload. J Clin Invest 109: 373-381, 2002.

23. Haq S, Choukroun G, Kang ZB, Ranu H, Matsui T, Rosenzweig A, Molkentin JD, Alessandrini A, Woodgett J, Hajjar R, et al: Glycogen synthase kinase-3beta is a negative regulator of cardiomyocyte hypertrophy. J Cell Biol 151: $117-130,2000$.

24. Creemers EE and Pinto YM: Molecular mechanisms that control interstitial fibrosis in the pressure-overloaded heart. Cardiovasc Res 89: 265-272, 2011.

25. Hutchinson KR, Guggilam A, Cismowski MJ, Galantowicz ML, West TA, Stewart JA Jr, Zhang X, Lord KC and Lucchesi PA: Temporal pattern of left ventricular structural and functional remodeling following reversal of volume overload heart failure. J Appl Physio 111: 1778-1788, 2011. 